\title{
Back-Stepping Control of Free-Floating Space Robot based on Adaptive Neural Network
}

\author{
Wang $\mathrm{Cao}^{1}$, Lin senhai ${ }^{2}$, Ye Xiaoping ${ }^{3}$, Jiang $\mathrm{Jie}^{4}$, Zhang Wenhui ${ }^{\star 5}$ \\ ${ }_{1,3,4,5}$ School of Engineering, Lishui University, Lishui 323000, China \\ ${ }^{2}$ Zhejiang Sikairui Robot Co., LTD, Lishui 323000, China -323000, Telp/Fax 0578-2271250 \\ *Corresponding author, e-mail: hit_zwh@126.com
}

\begin{abstract}
Trajectory tracking control problems of the free-floating space robot are considered by the paper, back-stepping control method based on adaptive neural network is put forward. The complex system is decomposed into several simple sub-systems. The control laws are designed by derived, so that closedloop stability can be obtained by each subsystem; Because of the influence of interference and the measurement level limitation, accurate mathematical model is difficult to be obtained. Neural network controller of good nonlinear approximation ability is designed to compensate the uncertainty of system model. Adaptive learning laws are designed to ensure that weights can be adjusted online real-time. The system uniformly ultimately bounded (UUB) is proved based on the Lyapunov theory. Simulation experiments show that the control method can fast track the desired trajectory, and has a good application value for space robotic manipulators with uncertainty.
\end{abstract}

Keywords: neural network, back-stepping control, space robot, adaptive control, uniformly ultimately bounde

Copyright @ 2017 Universitas Ahmad Dahlan. All rights reserved.

\section{Introduction}

Space robotic manipulators play an increasingly important role in space exploration, It can maintenance and recovery of satellite, construct the space station instead of astronauts on a dangerous mission, etc. Uncontrolled free-floating space robotic manipulators have more valuable, because it can save fuel control, increase the effective service life, and reduce launch costs [1-4].

Space robotic manipulators have different dynamic characteristics with the ground robot because of its space micro-gravity environment, including the dynamic coupling of base and manipulators, dynamic singular, limited fuel supply and restriction of the attitude control system. These factors lead to a strong nonlinear of space robot dynamics, therefore, the space robot dynamics and control problems are more complex than ground-fixed robot, the usual control strategy could not be adopted. At the same time, there are much uncertainty for space robot, for example, the dynamic model of manipulators, inertia matrix and load mass couldn't be obtained accurately and the external disturbance signals have certain bad influence to the controller [5-9].

To eliminate the influences of these nonlinear factors, the advanced control methods were applied to copy with control problems [10-14]. For example, adaptive control methods, back-stepping control methods, fuzzy control methods and neural network control methods, etc [15-18]. A neural network adjustment control strategy was proposed for the space robot with uncertainty by ref [19], the uncertain upper bound of nonlinear function was approximated by neural network controller. A feed-forward neural network control strategy was proposed by ref [20], the dynamic model was established by neural network controller. A neural network control strategy was proposed for the non-intact free-floating space robot system by ref [21], the adaptive control method was designed to adjust the network weights off-line. The compensation control method base on radial basis function (RBF) neural network was proposed by ref [18], the controller could approach nonlinear dynamic model of space robot, and the neural network parameters could be adjusted on line. A neural network adaptive controller was presented for multiple-input and multiple-output system by ref [23], robust controller was designed to eliminate the modeling errors, and the control method could achieve good control results. The application 
of adaptive neural-fuzzy control to the task-space trajectory following issue for mobile manipulators was proposed by ref [24]. Both tracking stability and error convergence could be guaranteed by the neural-fuzzy control system.

For the uncertainty model of the space robot, the paper proposes an adaptive neural network control algorithms base on back-stepping design. In case of fact condition, the controller precise mathematics model of space robot controller obtained hardly. The complex system is decomposed into several simple sub-systems. The control laws are designed by derived, so that closed-loop stability can be obtained by each subsystem. Uncertainty models are compensated by neural network controller. The weight adaptive control laws are designed to ensure adjustment online real-time. Uniformly ultimately bounded (UUB) of the whole closedloop system should be proved based on Lyapunov theory in the paper.

\subsection{Dynamic Equation of Space Robot}

Consider the single-arm space robotic manipulators model in Figure1 [18-21].

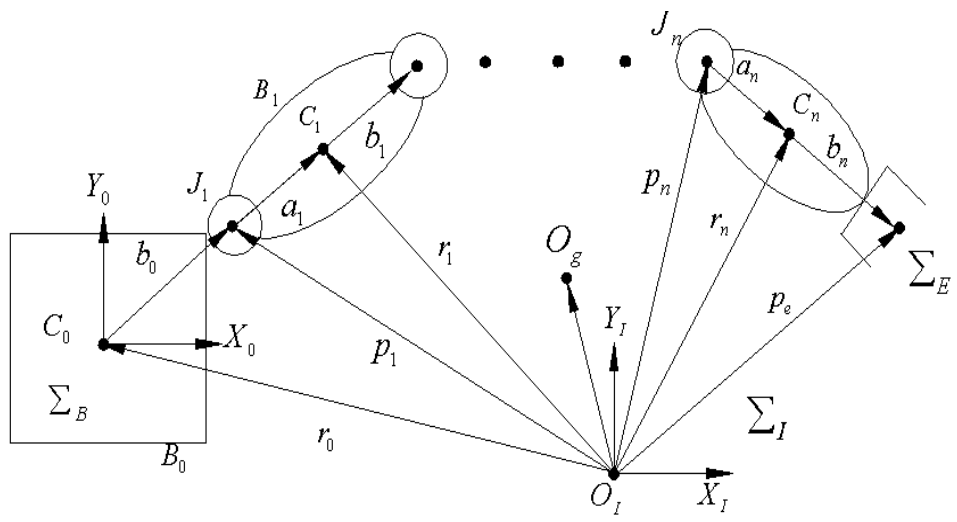

Figure 1. Parameters of Manipulators

Base of Space robotic manipulators in micro-gravity environment is fixed, so that the movement of whole system increases six degrees of freedom. The manipulators system with $n$ joints have $n+6$ degrees of freedom, movement of space robotic manipulators can produce a torque or force to the base, it makes position of the base change. Lagrange function is equal to the system kinetic energy [8], $\mathrm{n}$-joints space manipulators dynamics equation is obtained based on the Lagrange method [8-14]:

$$
M^{*}(q) \ddot{q}+C^{*}(q, \dot{q}) \dot{q}+d^{*}=\tau^{*}
$$

Where, $M^{*}(q) \in R^{(n+6)(n+6)}$ are inertia matrix of the robot system, $C^{*}(q, \dot{q}) \in R^{(n+6)(n+6)}$ are the centrifugal and Coriolis force, $q=\left[\begin{array}{ll}q_{b}^{T} & q_{m}^{T}\end{array}\right]^{T} \in R^{n+6}, q_{b} \in R^{6}$ are body posture angle, $q_{m}=\left[\begin{array}{llll}q_{1} & q_{2} & \cdots & q_{n}\end{array}\right]^{T} \in R^{n}$ are manipulator joint angle with $\mathrm{n}$-joints, $d^{*} \in R^{n+6}$ are bounded disturbances. $\tau^{*}=\left[\begin{array}{ll}\beta_{6 \times 1}^{T} & \tau_{n \times 1}^{T}\end{array}\right]^{T} \in R^{n+6}, \beta_{6 \times 1}$ are the output of control torque of the base., $\tau_{n \times 1}$ are the output of control torque on the manipulator joints.

Because the base of free-floating space robot cannot be controlled, the precious space energy can be saved, So $\beta_{6 \times 1}=0$.At the same time, in the system are not subject to any other external force and torque, the momentum conservation, the free-floating space robot dynamics equation as follows [15-21]:

$$
M\left(q_{m}\right) \ddot{q}_{m}+C\left(q_{m}, \dot{q}_{m}\right) \dot{q}_{m}+d=\tau
$$


Where, $M\left(q_{m}\right) \in R^{n \times n}$ are inertia matrix of the robot system, $C\left(q_{m}, \dot{q}_{m}\right) \in R^{n \times n}$ are the centrifugal and coriolis force, $d \in R^{n}$ are bounded disturbances. $\tau \in R^{n}$ are the output of control torque on the manipulator joints.

Free-floating space manipulators dynamics equation (2) has the following properties and hypothesis [16-22]

Property 1: Inertia matrix $M\left(q_{m}\right)$ is symmetric positive definite matrix, and bounded.

Property 2: Through appropriate selection of $C\left(q_{m}, \dot{q}_{m}\right), \dot{M}\left(q_{m}\right)-2 C\left(q_{m}, \dot{q}_{m}\right)$ could be skew symmetric matrix.

\section{Design of Neural Network Controller based on Back-Stepping}

Define $x_{1}=q_{m}, \dot{x}_{2}=\dot{q}_{m}$, assume $y=q_{m}$ is the output, so $\dot{x}_{1}=x_{2}, y=x_{1}$, the space robot dynamic equation (2), it could be written:

$$
\dot{X}_{2}=M^{-1}\left(x_{1}\right) \tau-M^{-1}\left(x_{1}\right) C\left(x_{1}, x_{2}\right) X_{2}-M^{-1}\left(x_{1}\right) d
$$

In case of the practice condition, the free-floating space robot dynamic model is obtained hardly; assume $M^{-1}\left(x_{1}\right) 、 C\left(x_{1}, x_{2}\right)$ are unknown nonlinear smooth functions.

Assume $y_{d}$ is the anticipated angle output, $y$ is the actual output, $y_{d}$ is command trajectory tracking.

The error is:

$$
z_{1}=y-y_{d}
$$

Assume $\alpha_{1}$ is the estimated value of $X_{2}$, define the error is:

$$
Z_{2}=x_{2}-\alpha_{1}
$$

By selecting $\alpha_{1}$, let $Z_{2}$ tend to 0 . Then:

$$
\dot{z}_{1}=\dot{x}_{1}-\dot{y}_{d}=x_{2}-\dot{y}_{d}=z_{2}+\alpha_{1}-\dot{y}_{d}
$$

The virtual control item is:

$$
\alpha_{1}=-\lambda_{1} z_{1}+\dot{y}_{d}
$$

Here, $\lambda_{1}>0$

For the first subsystem of equation (3), Lyapunov function is designed:

$$
L_{1}=\frac{1}{2} Z_{1}^{T} Z_{1}
$$

Then:

$$
\dot{L}_{1}=z_{1}^{T} \dot{Z}_{1}
$$


Bring equation (5) and (6) into the above:

$$
\dot{L}_{1}=-\lambda_{1} z_{1}^{T} Z_{1}+Z_{1}^{T} Z_{2}
$$

It is can be seen from the above equation, if $Z_{2}=0$, then

$$
\dot{L}_{1} \leq 0
$$

From equation (3) and (5), it can be educed:

$$
\dot{Z}_{2}=-M^{-1} C x_{2}-M^{-1} d+M^{-1} \tau-\dot{\alpha}_{1}
$$

The control law is:

$$
\tau=-\lambda_{2} z_{2}-z_{1}-\hat{f}
$$

Where, $\hat{f}$ is the item to be designed, $\lambda_{2}>0$.

\section{Proof:}

Lyapunov function is defined:

$$
L_{2}=L_{1}+\frac{1}{2} z_{2}^{T} M z_{2}
$$

Then,

$$
\dot{L}_{2}=\dot{L}_{1}+\frac{1}{2} z_{2}^{T} M \dot{z}_{2}+\frac{1}{2} \dot{z}_{2}^{T} M z_{2}+\frac{1}{2} z_{2}^{T} \dot{M} z_{2}
$$

Bring equation (9) into the above, then

$$
\dot{L}_{2}=-\lambda_{1} z_{1}^{T} z_{1}+z_{1}^{T} z_{2}+z_{2}^{T} M \dot{z}_{2}+\frac{1}{2} z_{2}^{T} \dot{M} z_{2}
$$

Bring equation (10) into the above and using the property 2.

Then:

$$
\begin{aligned}
& \dot{L}_{2}=-\lambda_{1} z_{1}^{T} z_{1}+z_{1}^{T} z_{2}+z_{2}^{T} M\left(-M^{-1} C X_{2}-M^{-1} d+M^{-1} \tau-\dot{\alpha}_{1}\right)+z_{2}^{T} C z_{2} \\
& =-\lambda_{1} z_{1}^{T} z_{1}+z_{1}^{T} z_{2}+z_{2}^{T}\left(-C \alpha_{1}-M \dot{\alpha}_{1}+\tau\right)-z_{2}^{T} d
\end{aligned}
$$

Bring the control law equation (11) into the above and arrange the equation,

$$
\dot{L}_{2}=-\lambda_{1} z_{1}^{T} Z_{1}-\lambda_{2} z_{2}^{T} Z_{2}+z_{2}^{T}\left(-C \alpha_{1}-M \dot{\alpha}_{1}-\hat{f}\right)-z_{2}^{T} d
$$

Considering the accuracy space robot model obtained hardly, the uncertainty need compensate to ensure closed-loop system stability.

The uncertainty part is $f=-C \alpha_{1}-M \dot{\alpha}_{1}$, considering of the better approximation ability of neural network, local generalization neural network is adopted to approach the uncertainty part $f$, and it could greatly accelerate the learning speed and avoid local minimum problems.

Assume $x$ is the output of the neural network, the item to be designed $\hat{f}$ is the estimated output of $f$, then: 


$$
\hat{f}=\hat{\theta}^{T} \varphi(x)
$$

Where, $\hat{\theta}$ is the actual neural network weight matrix, $\varphi(x)$ is Gaussian function, then $\theta_{1} \theta_{2}$ $\theta_{\mathrm{j}} \varphi_{1}(x) \quad \varphi_{2}(x) \quad \varphi_{j}(x)$

$$
\varphi_{j}=\exp \left(-\frac{\left\|x-c_{j}\right\|^{2}}{\sigma_{j}^{2}}\right)
$$

In the equations, $c_{j}$ is the center of the $\mathrm{j}$ basis function, $\sigma_{j}$ is the width of basis function, $\left\|x-c_{j}\right\|$ is the norm of vector $X-c_{j}$ assumptions.

According to approximation ability of RBF neural network, make the following

Assumption 1: For any given small positive number $\varepsilon_{d m}$, the optimal weight vector $\theta^{*}$ could always be found, let the approximation error meet $|\varepsilon|=\left|\theta^{* T} \varphi(x)-f\right|<\varepsilon_{d m}$.

Assumption 2: The optimal weigh vector $\theta^{*}$ is bounded, positive number $k$ exists, meeting $\left\|\theta^{*}\right\| \leq k$.

Then:

$$
f=\theta^{* T} \varphi(x)+\varepsilon
$$

The adaptive algorithm of neural network weight matrix is:

$$
\dot{\tilde{\theta}}=-\eta\left(z_{2}^{T} \varphi^{T}(x)\right)^{T}+2 k \theta
$$

Gain $k$ and $\eta>0, \tilde{\theta}=\theta^{*}-\hat{\theta}$ is the estimated error of weight.

\section{System Stability Analysis}

Define the following Lyapunov functions to prove closed-loop stability of free-floating space robot.

$$
L=L_{2}+\frac{1}{2} \tilde{\theta}^{T} \eta^{-1} \tilde{\theta}
$$

On both sides, take differential, then

$$
\begin{aligned}
& \dot{L}=z_{2}^{T}\left(f-\hat{\theta}^{T} \varphi(x)\right)-\lambda_{1} z_{1}^{T} z_{1}-\lambda_{2} z_{2}^{T} z_{2}-z_{2}^{T} d+\tilde{\theta}^{T} \eta^{-1} \dot{\tilde{\theta}} \\
& =z_{2}^{T}\left(f-\theta^{* T} \varphi(x)\right)+z_{2}^{T}\left(\theta^{* T} \varphi(x)-\hat{\theta}^{T} \varphi(x)\right)-\lambda_{1} z_{1}^{T} z_{1}-\lambda_{2} z_{2}^{T} z_{2}-z_{2}^{T} d+\tilde{\theta}^{T} \eta^{-1} \dot{\tilde{\theta}}
\end{aligned}
$$

Arrange the equation, then:

$$
\dot{L} \leq-\lambda_{1} z_{1}^{T} z_{1}-\lambda_{2} z_{2}^{T} z_{2}+\left\|z_{2}^{T}\right\| \cdot \|\left(f-\theta^{* T} \varphi(x)\left\|z_{2}^{T}\left(\tilde{\theta}^{T} \varphi(x)\right)-\right\| z_{2}^{T}\|\cdot\| d \|+\tilde{\theta}^{T} \eta^{-1} \dot{\tilde{\theta}}\right.
$$

Because of $2 a b \leq a^{2}+b^{2}$, adaptive control law equation (20) and assumption 1 , then 


$$
\dot{L} \leq-\lambda_{1} Z_{1}^{T} Z_{1}-\lambda_{2} Z_{2}^{T} Z_{2}+\frac{1}{2}\left\|z_{2}^{T}\right\|^{2}+\frac{1}{2} \varepsilon_{d m}^{2}+\frac{2 k}{\eta} \tilde{\theta}^{T} \theta+\frac{1}{2}\left\|z_{2}^{T}\right\|^{2}+\frac{1}{2}\|d\|^{2}
$$

Bring $\tilde{\theta}=\theta^{*}-\hat{\theta}$ into the above equation,

$$
\dot{L}=-\lambda_{1} Z_{1}^{T} Z_{1}-\lambda_{2} Z_{2}^{T} Z_{2}+Z_{2}^{T} Z_{2}+\frac{1}{2} \varepsilon_{d m}^{2}+\frac{1}{2} d^{T} d+\frac{2 k}{\eta}\left(\theta^{* T} \theta-\theta^{T} \theta\right)
$$

Considering of $\left(\theta-\theta^{*}\right)^{T}\left(\theta-\theta^{*}\right) \geq 0$, namely $2\left(\theta^{* T} \theta-\theta^{T} \theta\right) \leq-\theta^{T} \theta+\theta^{* T} \theta^{*}$, bring it to the above, then:

$$
\dot{L} \leq-\lambda_{1} Z_{1}^{T} Z_{1}-\lambda_{2} Z_{2}^{T} Z_{2}+Z_{2}^{T} Z_{2}+\frac{1}{2} \varepsilon_{d m}^{2}+\frac{1}{2} d^{T} d+\frac{2 k}{\eta} \theta^{* T} \theta^{*}+\frac{k}{\eta}\left(-\theta^{T} \theta-\theta^{* T} \theta^{*}\right)
$$

As $\left(\theta+\theta^{*}\right)^{T}\left(\theta+\theta^{*}\right) \geq 0$

So $-\theta^{* T} \theta-\theta^{T} \theta^{*} \leq \theta^{* T} \theta^{*}+\theta^{T} \theta$

Because $\tilde{\theta}=\theta^{*}-\hat{\theta}$

Then:

$$
\begin{aligned}
& \tilde{\theta}^{T} \tilde{\theta}=\left(\theta^{* T}-\theta^{T}\right)\left(\theta^{*}-\theta\right) \\
& =\theta^{* T} \theta^{*}+\theta^{T} \theta-\theta^{* T} \theta-\theta^{T} \theta^{*} \\
& \leq 2 \theta^{* T} \theta^{*}+2 \theta^{T} \theta
\end{aligned}
$$

Bring equation (27) to the equation (26), then:

$$
\dot{L} \leq-\lambda_{1} z_{1}^{T} Z_{1}-\lambda_{2} z_{2}^{T} Z_{2}+Z_{2}^{T} Z_{2}+\frac{1}{2} \varepsilon_{d m}^{2}+\frac{1}{2} d^{T} d+\frac{2 k}{\eta} \theta^{* T} \theta^{*}-\frac{k}{2 \eta} \tilde{\theta}^{T} \tilde{\theta}
$$

As $M$ is bounded, assume its boundary is $\sigma_{M} I$, and $\sigma_{M}>0$. If $\lambda_{2}>1$

Then:

$$
\dot{L} \leq-\lambda_{1} z_{1}^{T} Z_{1}-\left(\lambda_{2}-1\right) \frac{M}{\sigma_{M}} z_{2}^{T} Z_{2}+\frac{1}{2} \varepsilon_{d m}^{2}+\frac{1}{2} d^{T} d+\frac{2 k}{\eta} \theta^{* T} \theta^{*}-\frac{k}{2 \eta} \tilde{\theta}^{T} \tilde{\theta}
$$

Define $\mu=\min \left\{2 \lambda_{1}, 2\left(\lambda_{2}-1\right) / \sigma_{M}, k\right\}$

Then:

$$
\begin{aligned}
\dot{L} & \leq-\mu\left(\frac{1}{2} z_{1}^{T} z_{1}+\frac{1}{2} z_{2}^{T} M z_{2}+\frac{1}{2 \eta} \tilde{\theta}^{T} \tilde{\theta}\right)+\frac{1}{2} \varepsilon_{d m}^{2}+\frac{1}{2} d^{T} d+\frac{2 k}{\eta} \theta^{* T} \theta^{*} \\
& =-\mu L+\frac{1}{2} \varepsilon_{d m}^{2}+\frac{1}{2} d^{T} d+\frac{2 k}{\eta} \theta^{* T} \theta^{*}
\end{aligned}
$$

As disturbance $d$ is bounded, assume its boundary is $d_{M}$, then: 


$$
\dot{L} \leq-\mu L+\frac{1}{2} \varepsilon_{d m}^{2}+\frac{1}{2} d_{M}^{2}+\frac{2 k}{\eta} \theta^{* T} \theta^{*}
$$

If $\omega=\frac{1}{2} \varepsilon_{d m}^{2}+\frac{1}{2} d_{M}^{2}+\frac{2 k}{\eta} \theta^{* T} \theta^{*}$, the above equation could be written as:

$$
\dot{L}=-\mu L+\omega
$$

Solve the first order differential equations, then:

$$
\begin{aligned}
& L(t) \leq L(0) \exp (-\mu t)+\frac{\omega}{\mu}[1-\exp (-\mu t)] \\
& \leq L(0)+\frac{\omega}{\mu} \quad \forall t \geq 0
\end{aligned}
$$

$L(0)$ is the initial value of $L$, define concourse

$$
\Omega_{0}=\left\{X \mid L(X) \leq L(0)+\frac{\omega}{\mu}\right\}
$$

Then:

$$
\left\{Z_{1}, Z_{2}, \tilde{\theta}\right\} \in \Omega_{0}
$$

Because $L$ is bounded, so all signals of closed-loop system are bounded.

\section{Simulations}

Two link free-floating space robotic manipulators simulation model of planar motion is to verify the effectiveness of the proposed control strategy. Table 1 is the simulation parameters of two degree of freedom space robot.

Table 1. Parameters of Space Robotic Manipulators

\begin{tabular}{llll}
$m_{0}$ & $400 \mathrm{~kg}$ & $a_{1}$ & $0.75 \mathrm{~m}$ \\
$m_{1}$ & $10 \mathrm{~kg}$ & $a_{2}$ & $0.5 \mathrm{~m}$ \\
$m_{2}$ & $7 \mathrm{~kg}$ & $b_{0}$ & $0.5 \mathrm{~m}$ \\
$I_{0}$ & $66 \mathrm{~kg} \mathrm{~m}^{2}$ & $b_{1}$ & $0.75 \mathrm{~m}$ \\
$I_{1}$ & $1.5 \mathrm{~kg} \mathrm{~m}^{2}$ & $b_{2}$ & $0.5 \mathrm{~m}$ \\
$I_{2}$ & $0.5 \mathrm{~kg} \mathrm{~m}^{2}$ & & \\
\hline
\end{tabular}

Assume the unmodeled dynamics and external disturbance: $f=\left[q_{m 1} \dot{q}_{m 1} 0.2 \sin t\right.$, $\left.q_{m 2} \dot{q}_{m 2} 0.2 \sin t\right]^{T}$

The desired trajectory of two-joint space robot: $y_{1 d}=1.5+0.5(\sin 3 t+\sin 2 t)$; $y_{2 d}=1.5+0.5(\cos 2 t+\cos 4 t)$

Controller parameters of neural network: ${ }^{\varepsilon_{d m}}=0.5 ; \eta=10 ; k=1.2$. 
The initial value of the joint movement of base and manipulator: $q_{b}(0)=0, y_{1}(0)=3$, $y_{2}(0)=0$

The neural network initial weights are all 0 , the basic functions widths are 10 , the basis function centers are randomly selected in $0 \sim 1$. Hidden nodes are 50. Simulation results are shown in Figure 2-4. Figure 2 is the joint angle trajectory tracking curves; Figure 3 is unknown model and its neural network approach value, the approximation value is its norm; figure 4 is control torque of output.

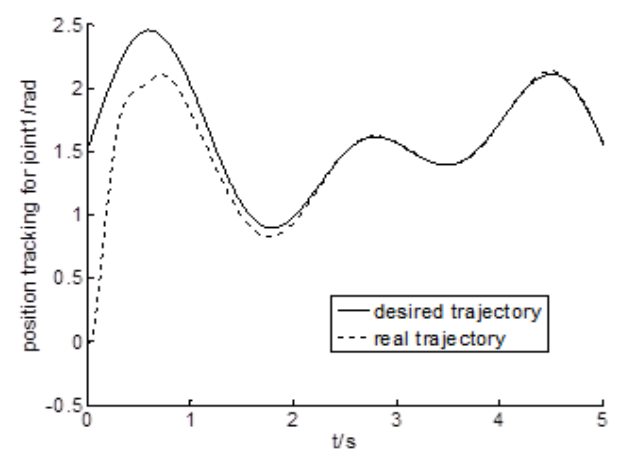

(a) Trajectory Tracking Curves of Joint 1

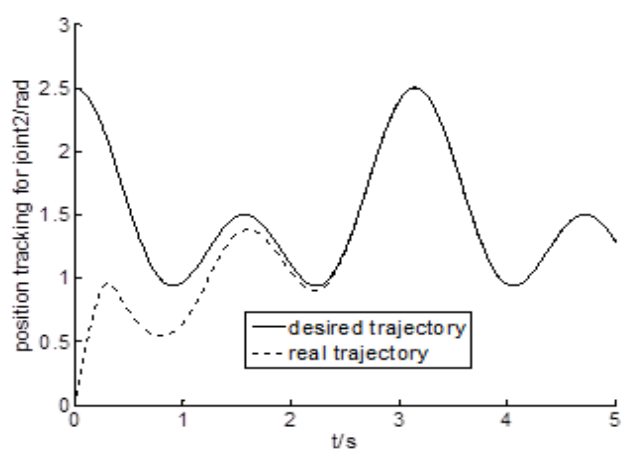

(b) Trajectory Tracking Curves of Joint 2

Figure 2. Trajectory Tracking Curves of Joins

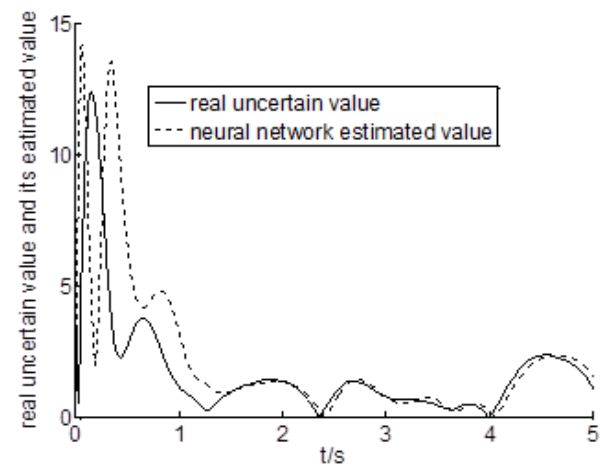

Figure 3. Unknown Model and Its Neural Network Approach

As can be found from Figure 2, even in the case the initial value error is bigger; the proposed neural network controller based on back-stepping could track the trajectory well in 2 seconds. Figure 3 shows that the neural network compensator could approach the space robot unknown model accurately in 1.5 seconds, it proves RBF neural network has not only the better approximation ability, but also the learning speed is fast. Figure 4 shows the control torque of the controller is not big.

To stay the attitude, the space robot usually works in the condition of low speed, the neural network has enough time to learn, and it could meet the performance requirement absolutely. 


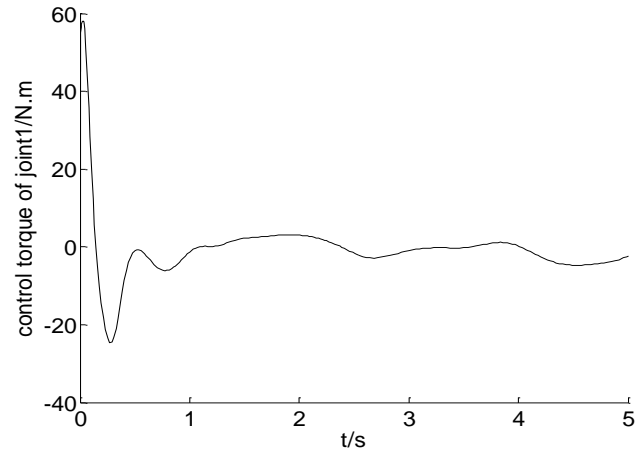

(a) Output Control Torque of Joint 1

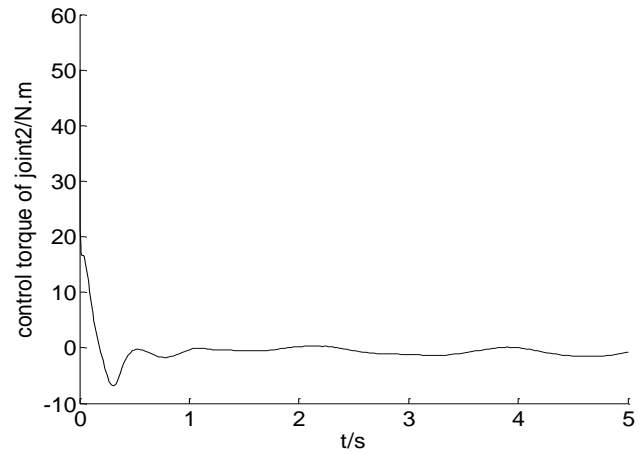

(b) Output Control Torque of Joint 2

Figure 4. Output Control Torque of Joins

\section{Conclusion}

Trajectory tracking problems of free-floating space robot with uncertainties are studied by the paper. An adaptive neural variable structure control algorithms base on back-stepping is proposed.

1. The complex system is decomposed into several simple sub-systems. The control laws are designed by derived, so that closed-loop stability can be obtained by each subsystem; Because of the influence of interference and the measurement level limitation, accurate mathematical model is difficult to be obtained.

2. Neural network controller of good nonlinear approximation ability is designed to compensate the uncertainty of system model. real-time

3. Adaptive learning laws are designed to ensure that weights can be adjusted online

4. Uniformly ultimately bounded (UUB) is proved based on the Lyapunov theory.

Simulation experiments show that the control method can fast track the desired trajectory, and has a good application value for space robotic manipulators with uncertainty.

\section{Acknowledgements}

This work was supported by Zhejiang Provincial Science and Technology Project (2015C31160), Zhejiang Province Education Department Project (Y201534043) and (Y201636936), Lishui Science and Technology Plan Project (2015KCPT03) and (2015RC04).

\section{References}

[1] GD King. Space Servicing: Past, Present and Future. In Proceedings of the 6th International Symposium on Artificial intelligence, Robot and Automation in space. Montreal, Canada. 2001.

[2] XP Hu, C Wang, WH Zhang, J Ma. Self-learning PID Control for X-Y NC Position Table with Uncertainty Base on Neural Network. TELKOMNIKA. 2014; 12(2): 343-348.

[3] S Dubowsky, EG Papadopoulos. The kinematics, Dynamics and control of free-flying space robotic systems. IEEE Transactions on Robotics and Automation. 1993; 9(5): 531-543.

[4] YM Fang, WH Zhang, XP Ye. Variable Structure Control for Space Robots Based on Neural Networks. International Journal of Advanced Robotic Systems. 2014; 11(3): 35-42.

[5] AK Bejczy, ST Venkataraman. Introduction to the special issue on space robotics. IEEE Transactions on Robotics and Automation. 1993; 9(5): 521-523.

[6] WH Zhang, XP Ye, LH Jiang, et al. Output feedback control for free-floating space robotic manipulators base on adaptive fuzzy neural network. Aerospace Science and Technology. 2013; 29(1): 135-143.

[7] L Chen. Adaptive and robust composite control of coordinated motion of space robot system with prismatic joint. In Proceedings of the 4th world Congress on Intelligent Control and Automation. Shanghai, China. 2002; 2: 1255-1259.

[8] XP Ye, WH Zhang, YM Fang. Neural Network Adaptive Control for X-Y Position Platform with Uncertainty. TELKOMNIKA. 2014; 12(1): 79-88. 
[9] CC Cheah, SA Kawamura, K Lee. Hळ Tuning for Task-Space Feedback Control of Robot with Uncertain Jacobian Matrix. IEEE Transactions on Automatic Control. 2001; 46(8): 1313-1318.

[10] YL Gu, YS Xu. A normal form augmentation approach to adaptive control of space robot systems. In Proceedings of the IEEE International Conference on Robotics and Automation. Atlanta, USA. 1993: 731-737.

[11] CK Lin. Non-singular terminal sliding model control of robot manipulators using fuzzy wavelet networks. IEEE Transactions on Fuzzy Systems. 2009; 160(12): 1765-1786.

[12] SD Wang, C K Lin. Adaptive control of robot manipulator using fuzzy compensator. Fuzzy Sets and Systems. 2000; 110(3): 351-363.

[13] $\mathrm{CH}$ Wang, CH Tsai, WS Lin. Robust fuzzy model-following control of robot manipulators. IEEE Transactions on Fuzzy Syst. 2000; 8(4): 462-469.

[14] CK Lin. Ho reinforcement learning control of robot manipulators using fuzzy wavelet networks. Fuzzy Sets and Systems. 2009; 160(12): 1765 -1786.

[15] FL Lewis, YH Kim. Intelligent optimal control of robotic manipulators using neural networks. Automatica. 2000; 36(9): 1355-1364.

[16] L Chen. Adaptive and robust composite control of coordinated motion of space robot system with prismatic joint. In Proceedings of the 4th world Congress on Intelligent Control and Automation. Shanghai, China. 2002; 2: 1255-1259.

[17] H Zhang, XP Ye, XM Ji, et al. RBF neural network adaptive control for space robots without speed feedback signal. Transactions of the Japan Society for Aeronautical and Space Sciences. 2013; 56(6): 317-322.

[18] YH Kim, FL Lewis. Neural network output feedback control of robot manipulators. IEEE Transactions on Robotics and Automation. 1999; 15(2): 301-309.

[19] J Xie, GL Liu, SZ Yan, WF Xu, WY Qiang. Study on Neural Network Adaptive Control Method for Uncertain Space Manipulator. Journal of Astronautics. 2010; 31(1): 123-129.

[20] RT Newton, Y Xu. Neural Network Control of a Space Manipulator. IEEE Control Systems Magazine. 1993; 13 (6): 14-22.

[21] WH Zhang, YM Fang, XP Ye. Adaptive Neural Network Robust Control for Space Robot with Uncertainty. TELKOMNIKA. 2013; 11(3): 513-520.

[22] BM Feng, GC Ma, WN Xie, CH Wang. Robust Tracking Control of Space Robot via Neural Network. Proceedings of 2006 Int.Symposium on Systems and Control in Aerospace and Astronautics. Harbin, China. 2006: 902-906.

[23] NS Edgar, Miguel AB. Adaptive recurrent neural control for nonlinear system tracking. IEEE Transactions on Systems, Man and Cybernetics, Part B: Cybernetics. 2000; 30(6): 886-889.

[24] Y Lin, Y Liu. Adaptive neural-fuzzy control for a nonholonomic mobile modular manipulator moving on a slope. Proc. IEEE Int. Conf. Mechatronics. Taipei, Taiwan, R.O.C. 2005: 358-363. 